\title{
A Review of Epilepsy Diagnosis Using PET Parameters
}

\author{
Yadalam Kiran Kumar ${ }^{1}$, Shashi Bhushan Mehta ${ }^{1}$, Uday Patil ${ }^{2}$ \\ ${ }^{1}$ Philips Electronics India Ltd, Bangalore, India \\ ${ }^{2}$ Manipal Hospital, Bangalore, India \\ Email: Kiran.kumary@philips.com, shashi.mehta@philips.com, Uday.Patil@philips.com
}

Received February 10, 2011; revised March 20, 2011; accepted April 5, 2011

\begin{abstract}
Epilepsy is caused by abnormal excessive electric discharges from the neurons in the brain. Epileptic seizures are nonspecific responses of the brain to many types of insults. The structural abnormalities causing epilepsy can be identified using various state of art imaging methods. Through a combination of brain activity monitoring, imaging and mapping techniques, physicians can locate the specific area in the brain causing epileptic discharges and identify its location in relation to those areas in the brain controlling vital functions. Positron Emission Tomography (PET) has emerged as a useful tool to identify abnormal metabolic activity of the epileptogenic foci. Parameters like asymmetric index, standard uptake value (SUV) etc. obtained by PET are processed and analyzed for identifying the origin of epileptic seizures. This paper discusses the techniques used to diagnose in general and to localize the epileptogenic regions using post-processing other features on PET imaging.
\end{abstract}

Keywords: Epilepsy; PET; Asymmetric Index; Standard Uptake Value

\section{Introduction}

Epilepsy is a chronic neurological disorder characterized by seizures. An epileptic seizure is a transient symptom of excessive or synchronous neuronal activity in the brain. Seizures may be provoked by acute events including infection, head injury, electrolyte imbalance, and stroke or brain tumors. Seizures are sudden alterations in behavior or motor function caused by an electrical discharge from the brain. Seizures can manifest as involuntary changes in body movement or function, sensation, awareness, behavior loss of consciousness, short or long term memory loss, involuntary contraction of a groups of muscles, or numbness of a part of the body [1].

Seizures result from electrical activity in any part of the brain and can therefore present in many different ways. They are broadly classified as either generalized or partial seizures $[1,2]$. Generalized seizures involve both halves of the brain simultaneously and are sometimes associated with loss of consciousness. Seizures can be of many types, including: tonic-clonic seizures: the body stiffens and then jerks repeatedly (old term: grand mal epilepsy); absence seizures: the person stares blankly for a few seconds (old term: petit mal epilepsy); myoclonic seizures: involves the abrupt jerking of muscle groups. In their severe form they can throw the individual to the ground; atonics seizures: involves a sudden loss of posture or tone in limbs or the whole body [2].
Partial seizures start in a localized part of the brain and spread from this point. They produce symptoms relating to that part's function such as motor, sensory, autonomic or psychic symptoms and may or may not be associated with a loss of consciousness. Unexplained muscle jerks in a limb, smells, flushing or pallor, or sensations of fear or pleasure may, when they precede a seizure (an aura), be clues as to the origin in the brain of that seizure. Partial seizures can be simple partial seizures-consciousness is not impaired, or complex partial seizures-consciousness is impaired. Epilepsy and its various types are evaluated by various medical tests and imaging techniques. These can be history and physical examination, and laboratory tests. The Imaging techniques include MRI or CT or Nuclear scans.

\section{Epilepsy and Its Diagnosis \& Tools}

The physicians start the process of diagnosis with the medical history and physical examination. This is followed by laboratory tests and Electroencephalography (EEG). EEG is electrical recording from multiple points from the scalp of the head and displays graphs of the electrical activity from these pints. The examination during the seizure or during first few hours after seizure is vital to accomplish correct diagnosis and localization. Patient history and examination helps to correctly iden tify the type of seizure, and at times, the cause, and the possible location of abnormality in the brain [3]. 


\subsection{Medical History}

Here the goal of medical history is to determine whether the event was truly a seizure. The Neurologist needs to know when the seizures started, and have a detailed description of the seizures. The family's health history is also considered. Witnessing the seizure is the best way to classify the type of epilepsy. Several drugs are also known to cause epileptic seizures. The history also focuses on the risk factors and predisposing events. In many cases the diagnosis of a seizure is based on the clinical grounds - the examination and the laboratory studies may not reveal any abnormality.

\subsection{Physical Examination}

The clinical examination is directed toward the identification of the underlying factors and adequacy of the patient current therapy. The general physical examination includes a search for signs of infection or systemic illness. Careful examination of the skin may reveal signs of neurocutaneous disorders, such as tuberous sclerosis which can cause intractable epilepsy. All the patients require a complete neurologic examination, with particular emphasis on eliciting signs of cerebral hemispheric disease which may be a pointer for the location and cause of epilepsy.

\subsection{Laboratory Studies}

The laboratory tests are performed to check for biochemical and toxicological abnormalities resulting in abnormal electrolytes, glucose, calcium or magnesium, and hepatic or renal dysfunction. Some poisons could also precipitate seizures [4].

\subsection{Lumbar Puncture}

Sometime lumbar puncture is also required to rule out any infection in the central nervous system. In this procedure, a needle is inserted in the lower back in order to gather the cerebrospinal fluid that surround the brain and spinal cord.

\subsection{Electroencephalogram (EEG)}

EEG is used for diagnosing the type of seizures and their location in the brain. This can be invasive or non-invasive to detect and record electrical impulses on the surface of the brain. In the non-invasive EEG, the impulses are recorded from small metal discs placed on the person's scalp [8]. The EEG recording is analyzed by a neurologist to determine whether there are any irregular electrical activities occurring in the brain. It can help identify the location, severity, and type of seizure disorder. An abnormal EEG does not always confirm epilepsy, nor does a normal EEG reading exclude it. Fewer than
$50 \%$ of routine EEGs are abnormal in patients who are known to have epilepsy [5].

Video-EEG is a prolonged recording of EEG along with video monitoring of the patients activities, allows a more thorough analysis of the EEG at the time of epileptic events, thereby increasing the likelihood of capturing epileptiform discharges. This analysis also is aided by the use of automated spike detection [6].

Invasive EEG is necessary when surgery is being considered. It is performed to define the zone of seizure activity with high precision in the brain cortex. Various techniques, each having advantages and limitations, are available, including subdural, epidural, foramen ovale and intra-cerebral (depth) electrodes.

- Instrument for recording electrical activity of brain.

- Generates in the individual neuron of the brain.

- Effective method for diagnosing many neurological illness and diseases such as epilepsy, tumor, sleeping patterns, mental disorders, etc.

- Electrodes around the head (scalp or cerebral cortex) measures and records activity of the brain.

EEG Electrodes:

Electrodes are a conductor, not necessarily metallic, through which a current enters or leaves a nonmetallic medium, as an electrolytic cell, arc generator, vacuum tube, or gaseous discharge tube.

- Transform ionic currents from cerebral tissue into electrical currents used in EEG preamplifiers.

- Types of electrodes:

$\checkmark$ Scalp.

$\checkmark$ Sphenoidal.

$\checkmark$ Nasopharyngeal.

$\checkmark$ Electrocardiographic.

Various types of cup electrodes are as follows:

- Silver plated.

- Reusable.

- Center hole in cup allows for easy fill of electrode gel after adhesion of cup to scalp.

- Size: Adult-10 mm diameter; Pediatric- $6 \mathrm{~mm}$ diameter.

- Most widely used electrodes. The various frequencies of EEG signal are alpha, beta, gamma, and delta are shown in Figure 1. Alpha Waves:

- Alpha rhythm is one of the principle components of EEG.

- Indicator of state of alertness of the brain.

- Less than $10 \mu \mathrm{V}$ peak to peak.

- Arise from posterior brain in waking person with eyes closed.

Beta Waves:

- Less than $20 \mu \mathrm{V}$ peak to peak.

- Spreads over the entire brain, most predominant over the central region at rest. 


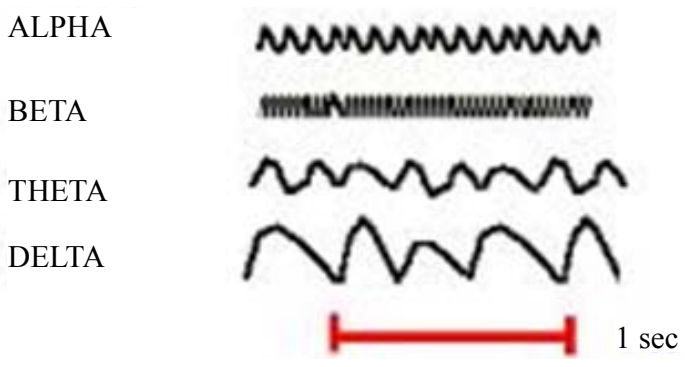

Figure 1. The various frequencies of EEG signal.

- High states of wakefulness and de-synchronized alpha patterns produce beta waves.

Delta Waves:

- The frequency is in the range of $3 \mathrm{~Hz}$ or less than 3 $\mathrm{Hz}$.

- Less than $30 \mu \mathrm{V}$ peak to peak. Gamma Waves:

- The frequency is in the range of $22-30 \mathrm{~Hz}$.

- Less than $35 \mu \mathrm{V}$ peak to peak.

\subsection{Magneto Encephalography (MEG)}

Magneto encephalography, also called Magnetic Source Imaging, is a non-invasive technique which provides information about function of the brain. It detects small biomagnetic signals produced by the brain. A map of this activity is created and co-registered with anatomic images obtained, typically from MRI. These signals provide information about the location of active brain areas. This technique allows the study of how different areas of the brain interact with one another [7]. MEG can help to identify brain zones which emit abnormal electric currents associated with epilepsy. It is useful in planning surgical treatment of epilepsy and for pre-surgical functional mapping of the brain [8].

\subsection{Wada Testing}

The Wada test simulates epilepsy surgery by putting one side of the brain to sleep for a short time and leaving the other side awake and functioning. With one side of the brain asleep, patients are given a series of tests that demonstrate speech and memory skills. The test is repeated on the other side to give doctors insight on where speech and memory reside in relation to the epileptic tissue. The Wada test helps to determine if a patient is a good candidate for epilepsy surgery or if the epileptic tissue is located in a critical area of the brain.

\section{Neuro Imaging in Epilepsy}

The understanding and treatment of epilepsy has been enormously improved by the development and use of brain-imaging techniques [9-11]. They provide information about the structure and function of the brain. As of date, there are various imaging modalities and integrated modalities such as Computed Tomography (CT), Magnetic Resonance Imaging (MRI), Single Photon Emission Computed Tomography (SPECT), and Positron Emission Tomography (PET). Almost all the patients with newonset seizures have a brain imaging study to determine the underlying structure abnormality. CT gives the structural details of the brain but MR imaging gives more exquisite details of structural abnormalities in the brain associated with epilepsy. The fundamental role of MRI in epilepsy imaging is structural evaluation, providing anatomical and pathologic information. Yet, not all causes can be detected with conventional MR imaging. Functional imaging comes into play for the remainder of the patients, which consists of radionuclide imaging (PETCT and SPECT). Advanced MR imaging techniques (Magnetic Resonance Spectroscopy (MRS), Diffusion Tensor Imaging (DTI), Functional MRI (fMRI) can also provide functional information.

\subsection{Computerized Axial Tomography}

CT (Computed Tomography) imaging is a non-invasive procedure which uses X-rays to create a computer-generated, three-dimensional image of the brain. Computed Tomography (CT) involves the data collection, image reconstruction and image display (image manipulation, storage, recording and communication) of cross-sectional anatomy. A broad summary of CT operations are as follows: the patient is positioned within the scanner. The $\mathrm{X}$-ray tube and detectors are internal to the scanner gantry and rotate around the patient during scanning. X-rays are attenuated as they pass through the patient and subsequently measured by the detectors. The detectors convert the X-ray photons into electrical signals; these are then converted into digital data for input to the computer. Image reconstruction is performed and then converted back into electrical signals for viewing on a monitor. The final images can be stored on digital media like hard disk, magnetic tapes, and optical disks and/or printed on X-ray film. These may reveal abnormalities (blood clots, cysts, tumors, scar tissue, etc.) in the skull or brain which may be related to seizures [12]. CT is most useful in evaluation of epileptic seizures which occurs in head injury. Its utility in other situations is much more limited and MRI is the imaging method of choice.

\subsection{Magnetic Resonance Imaging (MRI)}

MRI is based on the nuclear magnetic resonance (NMR) phenomenon first observed by Bloch and Purcell in 1946. NMR principles have made high quality image recovery possible by allowing the encoding of spatial information into signals that can be detected outside the body. MRI is a non-invasive imaging technique that uses a magnetic 
field, radio waves, and a computer to produce two or three dimensional images of the brain. These detailed images of brain structures helps physicians to locate possible causes of seizures and identify areas that may generate seizures.

Clinical Magnetic Resonance Imaging (MRI) uses the magnetic properties of hydrogen and its interaction with both a large external magnetic field and radio waves to produce highly detailed images of the human body. We derive the images from the magnetic resonance properties of nuclear particles (specifically hydrogen). In vivo MR imaging obtains resonance signal limited to intracellular and extracellular water and some lipids. The resonance signal is displayed as function of frequency spectrum converted into gray-scale values displayed as an image. In order to perform MRI, we first need a strong magnetic field. The field strength of the magnets used for MR is measured in units of Tesla. One (1) Tesla is equal to 10,000 gauss. The magnetic field of the earth is approximately 0.5 Gauss. Given that relationship, a $1.0 \mathrm{~T}$ magnet has a magnetic field approximately 20,000 times stronger than that of the earth. The type of magnets used for MR imaging usually belongs to one of three types; permanent, resistive, and superconductive. MRI has exquisite soft tissue resolution and hence allows identification of structural abnormalities and malformations in the brain. However, not all epileptics would have abnormal MRI $[13,14]$.

\subsection{Functional MRI}

The role of functional MRI (fMRI) in the presurgical evaluation of patients with intractable epilepsy is being increasingly recognized. Real-time fMRI is an easily performable diagnostic technique in the clinical setting. It has become a noninvasive alternative to intraoperative cortical stimulation and the Wada test for eloquent cortex mapping and language lateralization, respectively.

\subsection{Magnetic Resonance Spectroscopy/Magnetic Resonance Spectroscopic Imaging}

MR spectroscopy provides a measure of brain chemistry. The most common nuclei that are used are $1 \mathrm{H}$ (proton), 23Na (sodium), 31P (phosphorus). Proton spectroscopy is easier to perform and provides much higher signalto-noise than either sodium or phosphorus. Proton MRS can be performed within $10-15$ minutes and can be added on to conventional MR imaging protocols. It can be used to serially monitor biochemical changes in tumors, stroke, epilepsy, metabolic disorders, infections, and neurodegenerative diseases. The MR spectra do not come labeled with diagnoses. They require interpretation and should always be correlated with the MR images before making a final diagnosis. In general in vivo MR spectroscopy obtains resonance signal from molecules in tissue and cells. This signal can be displayed as spectral plot or MR spectroscopic data displayed in gray and color scale to produce a spectroscopic or chemical shift image, as in a study of patient with epilepsy. MR spectroscopy is a diagnostic tool, which has ability to noninvasively detect biochemical and their changing concentrations, which correlate with physiologic and pathological process [15].

Proton MRS can quantify neuronal loss. $\mathrm{N}$-acetyl acetate (NAA) is present in mature neurons and is the surrogate measure for neuronal dysfunction or loss. MRS can also measure gamma aminobutyric acid (GABA) receptor function in vivo. 1H (hydrogen) and 31P (phosphorus) spectroscopy detects metabolite changes for $\mathrm{Pa}$ tients suffering with temporal lobe epilepsy. Study shows reduction in NAA and in the ratio of $\mathrm{PCr} / \mathrm{Pi}$ using single voxel or chemical shift imaging [15-18].

\subsection{MR Image Analysis}

MR imaging used widely in neuro imaging as diagnostic tool gives good soft tissue contrast and the contrast depends on different parameters like magnetization relaxation time, proton density, diffusion, perfusion etc. But the intensity level variation from one tissue type to other is slow due partial volume effect, tissue variations or imaging sequences used during data acquisition, which opens opportunity in post processing and image analysis to improve the diagnostic value. The most commonly techniques in post processing are statistical or soft computing based applied on MR images [19-25]. Here voxels or pixels in an image are analyzed using different features like intensity, neighboring pattern, or geometric features to improve image quality. These techniques help in segmentation, image enhancement or registration [2628]. These post processing techniques helps to characterize tissue to assess disease area, location or monitor therapy response in general or epileptic cases.

\subsection{Angiography}

Its use is limited in epilepsy, except in vascular abnormalities and to perform the sodium amytal test (WADA) to determine language lateralization in selected patients for epilepsy surgery [29].

\subsection{SPECT (Single Photon Emission Tomography)}

Functional imaging, with both PET and single-photon emission CT (SPECT), has been widely useful in the diagnosis, management, and follow-up of patients with seizure disorders [30-33]. The ability of functional imaging to provide important information about seizures 
derives from the fact that epileptic conditions result in significant physiologic alterations in the brain. These physiologic changes occur both during seizures (ictus) and in the interictal state. Because generalized seizures affect a large part of the brain, it is typically more difficult to isolate the originating focus from other areas that are secondarily affected on functional imaging studies. For partial seizures and other types of seizures that originate from a specific focus, however, functional imaging can be useful for localizing the primary site. Functional imaging also helps in the understanding of the pathophysiology of seizure disorders $[34,35]$. SPECT is widely used in the localization of epileptogenic focus.

A SPECT Camera consists of a gamma camera or a set of gamma cameras mounted on a gantry so that the detector can record projections from many equally spaced angular intervals around the body. A coordinate system ( $r$, s) helps to determine how radioactivity at a location $(\mathrm{x}, \mathrm{y})$ in the object contributes to the signal recorded at location $r$ in the projection acquired at rotation angle $\varphi$. A Sinogram is a representation of the full set of projection data in the form of a 2-D matrix $\mathrm{p}(\mathrm{r}, \varphi)$. Each row across the matrix represents an intensity displayed across a single projection. The successive rows represent successive projection angles. There are different techniques to reconstruct topographic images.

Simple back projection - its general purpose is to generate $2 \mathrm{D}$ cross sectional image of activity from a slice within the object, using the projection profiles obtained for that slice. In the back projection the counts recorded in a particular projection profile element are divided uniformly amongst the pixels that fall within its projection path. When the back projections for all profiles are added together, an approximation of the distribution of radioactivity within the scanned slice is obtained. The reconstruction of the simple back-projection results in low quality images. This is the reason why the filtered back-projection is commonly used.

The ictal and interictal SPECT studies are used to localize the epileptogenic focus. The active epileptogenic area shows increased uptake during the ictus (during the seizure) and decreased uptake of the isotope in the interictal (between two seizures) state. Functional scanning with SPECT identifies the location of an epileptic focus in a substantial proportion and it is useful to improve the selection of patients for surgery.

\section{Positron Emission Tomography (PET)}

PET imaging has been used in the management of patients with seizure disorders over the past two decades. During an epileptic seizure, cerebral metabolism and cerebral blood flow are markedly increased in the epileptogenic focus. During the interictal period, both cere- bral metabolism and cerebral blood flow are decreased in this region, interictal fluorine-18-fluorodeoxyglucose (18F-FDG) PET studies have revealed no focal areas of hypo metabolism. The focus of partial seizures (with or without secondary generalized seizures) can be identified using FDG-PET, however, because the seizure foci have increased metabolic activity during the seizure and decreased metabolic activity between seizures [36,37]. It has been shown that single hypo metabolic regions can be identified in $55 \%$ to $80 \%$ of patients with focal surface electroencephalography (EEG) abnormalities. These areas of decreased metabolism often appear more extensive in size than do anatomic abnormalities observed on MR imaging. Ictal PET is difficult to perform as the availability of FDG at the time of the seizure cannot be ensured in view of its short half life [38].

\subsection{Working with Positron Emission Tomography}

PET is a non invasive method of nuclear imaging that uses short life radio-pharmaceuticals to detect and assess perfusion and metabolic activity in various organ systems. It provides information about function and metabolism that is complementary to the structural information provided by cross sectional imaging techniques such as MRI and CT. PET uses radioisotopes which decay by emitting a positively charged electron (positron) from the nucleus. The positron annihilates a negatively charged electron, resulting in two high-energy photons (511 kilo-electronvolts, $\mathrm{keV}$ ) that travel at 180 degrees to each other. The high-energy photon is subject to less absorption or scatter by tissue than the comparatively lower-energy photons released during conventional nuclear medicine imaging.

In order to detect the coincidences, the geometry of the PET detectors is the form of the ring as shown in Figure 2. Several parallel rings form the complete detection panel of the system. A coincidence event is assigned to a line of response (LOR) joining the two relevant detectors. In this way, positional information is gained from the detected radiation without the need for a physical collimator. This is known as electronic collimation. Electronic collimation has two major advantages over physical collimation. These are improved sensitivity and improved uniformity of the point source response function. Coincidence events in PET can be of four kinds: true, scattered, random and multiple [39].

True coincidences occur when both photons from an annihilation event are detected by detectors in coincidence, neither photon undergoes any form of interaction prior to detection, and no other event is detected within the coincidence time-window as shown in Figure 3. A scattered coincidence is one in which at least one of the detected photons has undergone at least one Compton 
scattering event prior to detection. Since the direction of the photon is changed during the Compton scattering process, it is highly likely that the resulting coincidence event will be assigned to the wrong line of response. Scattered coincidences add a background to the true coincidence distribution which changes slowly with position, decreasing contrast and causing the isotope concentrations to be over estimated. They also add statistical noise to the signal. The number of scattered events detected depends on the volume and attenuation characteristics of the object being imaged, and on the geometry of the camera.

Random coincidences occur when two photons not arising from the same annihilation event are incident on the detectors within the coincidence time window of the system. The number of random coincidences in a given LOR is closely linked to the rate of single events measured by the detectors joined by that LOR and the rate of random coincidences increase roughly with the square of the activity in the FOV. As with scattered events, the number of random coincidences detected also depends on the volume and attenuation characteristics of the object being imaged, and on the geometry of the camera. The

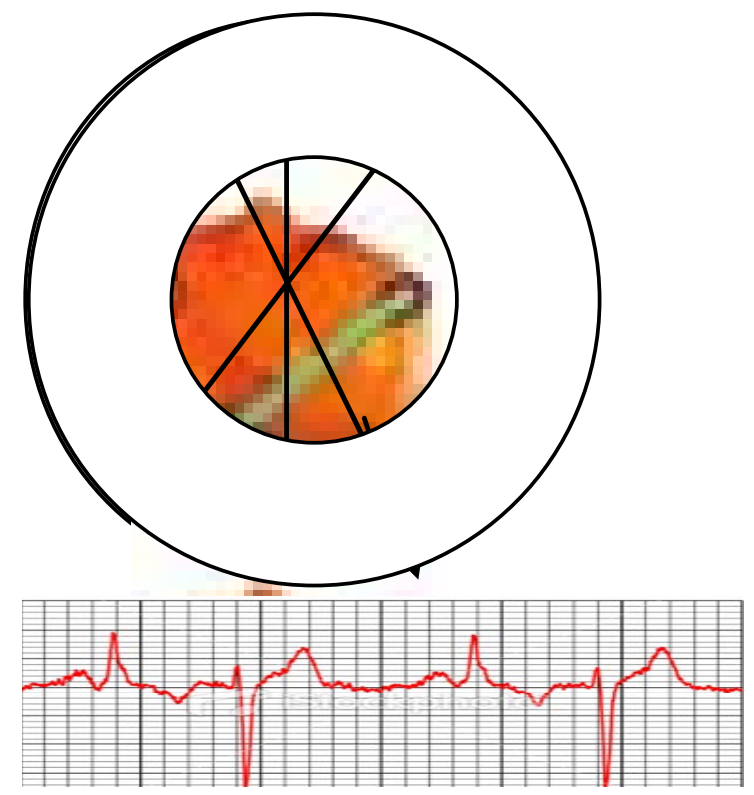

Figure 2. Coincidence detection in a PET camera.

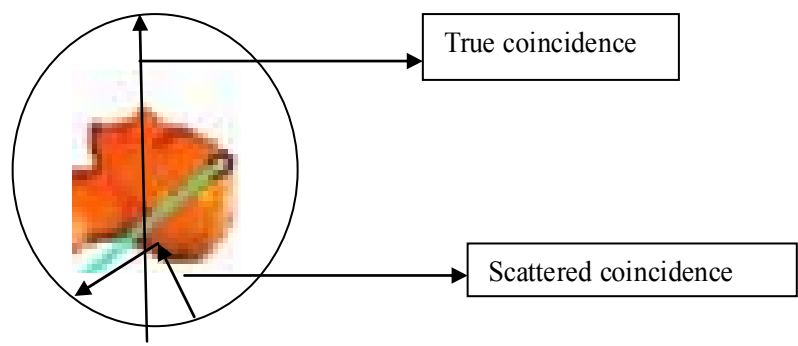

Figure 3. PET coincidence kinds. distribution of random coincidences is fairly uniform across the field of view, and will cause isotope concentrations to be overestimated if not corrected for. Random coincidences also add statistical noise to the data [40].

Most cameras employing block-detector technology may be operated either in " $2 \mathrm{D}$ " mode or " $3 \mathrm{D}$ " mode. In 2D mode thin septa of lead or tungsten separate each crystal ring and coincidences are only recorded between detectors within the same ring or lying in closely neighboring rings. Coincidences between detectors in closely neighboring rings are summed or rebinned to produce a dataset consisting of $2 \mathrm{P}+1$ co-planar sets of LORs normal to the axis of the camera, where $\mathrm{P}$ is the number of detector rings. Such a dataset may be reconstructed into images using tomographic techniques like the ones discussed by SPECT.

Corrections for scatter, random and the effects of attenuation are applied to data acquired in a PET camera then the number of counts assigned to an LOR joining a pair of crystals is proportional to a line integral of the activity along that LOR. Parallel sets of such line integrals are known as projections. Reconstruction of images from projections is a problem to which much attention has been paid over the last 30 years, and many analytical and iterative reconstruction schemata exist on in the computational burden [add some references for reconstruction algorithm]. One way to get attenuation correction is by running a transmission scan. It uses a radioactive source to produce an attenuation "map" of the body of the patient. A reference scan called "blank" is always done in order to obtain the ratio of the blank counts that is used for corrections and quality assurance with its pair, which usually results in superior image quality (Lewellen, Miyaoka \& Swan 1999, Why attenuation correction is required and any link or references related with epilepsy diagnosis).

The standard measurement of the attenuation correction method (with cesium-137 single-photon sources) was applied, in which the attenuation correction matrix is calculated by forward projection at appropriate angles of the resulting transmission image. The generated attenuation correction map is then used to reconstruct the emission data. The images were scatter-corrected and reconstructed by using normalized attenuation-weighted, ordered subset-expectation maximization iterative reconstruction. The default parameters used in clinical routine are ordered subset-expectation maximization iterative reconstruction with two iterations and eight subsets followed by a post processing Gaussian filter (kernel fullwidth half-maximal height, $6.0 \mathrm{~mm}$ ). The voxel size was set to $3.4 \times 3.4 \times 3.4 \mathrm{~mm}^{3}$ [41] .

\subsection{The Procedure}

Patients are asked to fast for five to six hours on the day 
of their scan. Upon arrival, FDG is injected via an intravenous cannula placed in the patient's arm. After the injection of FDG the patient waits up to an hour before undergoing the scan.

The analytic techniques range from simple visual qualitative assessment and semi quantitative methods (e.g. standardized uptake value (SUV) measurement), to full kinetic analysis with compartmental modeling (e.g. nonlinear regression analysis (NLR)) [42].

\section{Parameters for Epilepsy Diagnosis Using PET}

Positron Emission Tomography system is a well accepted modality to diagnose epileptic seizures causes and location by analyzing different parameters listed below. These parameters on image data are analyzed with various data processing tools, which include asymmetry, SUV statistical analysis, and Image enhancement, postprocessing of image data, besides image visualization in 2D and 3D mode. Various PET parameters [32-37] used to diagnose the epileptic patients are as follows:

- Cerebral blood flow measured with [15O] $\mathrm{H}_{2} \mathrm{O}$.

- Uptake of [18F] fluorodeoxyglucose (FDG), an index of the cerebral metabolism rate of glucose.

- Distribution volume (DV) of [11C] flumazenil (FMZ), an index of the binding potential of central benzodiazepine receptor.

- Standard uptake value.

\subsection{The Cerebral Blood Flow Measurement}

Cerebral blood flow is the blood supply to the brain in a given time. The variation of these parameters forms the basis to analyze the asymmetric index of the brain in the Left and Right hemisphere. The regional cerebral blood flow determines the amount of variation of the asymmetry in left and right hemisphere. This variation helps to find the level of asymmetries in the human brain. The various studies has been done in the literature such as by using the 11C labeled 1-(3,4-dimethoxyphenethyl)-4(3-phenylprophyl) piperazine (11C-SA4503) [43].

\subsection{Uptake of [18F] Fluorodeoxyglucose (FDG), an Index of the Cerebral Metabolism Rate of Glucose}

The tracer [18F]-fluorodeoxyglucose (FDG) used for measuring metabolic rate of glucose in the human brain for the epileptic patients in the Positron Emission Tomography (PET) analysis. The cerebral metabolism rate of the glucose helps to determine the amount of variation of the asymmetry in left and right hemisphere of the brain. The amount of absorption of the glucose in the brain determines the amount of activity in the left and right hemisphere of the brain, which is used for asymmetry analysis.

\subsection{Distribution Volume (DV) of [11C] Flumazenil (FMZ), an Index of the Binding Potential of Central Benzodiazepine Receptor}

The parameter for epilepsy diagnosis is by using distribution volume (DV) images. Various researches have been made in the literature to obtain standardized uptake value (SUV) images compatible with DV images. The use of [11C] flumazenil (FMZ) is useful to find the principal inhibitory neurotransmitter in the brain which helps to find SUV. There are various mathematical analysis and models in the literature for the identification of the neuronal activity in patients helps to find the asymmetry index variation of the brain for the epileptic patients [44].

\subsection{Standard Uptake Value (SUV)}

The SUV depends on the amount of injected radioactivity, the patient's weight, and the calibration factor of the camera and it is calculated according to the following formula

$\mathrm{SUV}=$ (radioactivity concentration in tissue $) /($ Injected dose) (Bq)/Patient weight (gms)

To calculate the SUV, images were reviewed, and the slice containing the tumor was selected. The degree of suspicion for malignant involvement was based on the qualitative visual interpretation of the images and the determination of the SUV, a semi quantitative measurement of relative FDG uptake within the regions of interest (ROIs). ROIs were defined over the whole tumor volumes as displayed on the different slices.

The drawback of SUV is that it is $20 \%$ variable for the phantom itself, hence it is not taken as the standard parameter for the epilepsy diagnosis and analysis for the patients. To overcome this issue, we use another parameter called asymmetric index [45].

\subsection{Calculation of Asymmetric Index for Intra Subject Variation}

Asymmetry in the brain in-case epilepsy detection plays an important role in diagnosis and localizing regions. To calculate the asymmetry index, we require the prior information such as the registration of images (left and right portion of brain), detection of midline in the brain and the finally for the calculation of the asymmetric index of the intra subject variation [46].

\subsubsection{Medical Image Registration}

The goal of image registration is to align, or spatially normalize, one image to another. In multi-subject studies, 
this reduces subject-specific anatomic differences by deforming individual images onto a population average brain template. Image registration is defined as the aligning of medical images with respect to the reference image. Image registration is obtained by placing the manual points in the location of left and right hemisphere and registers it by using rigid/non-rigid registration. The purpose of image registration is to align the left and right hemisphere and it is useful to calculate the asymmetry index of the brain. The literature shows various registration techniques to calculate the asymmetry of the brain for the epileptic images [47].

\subsubsection{Detection of Midline in Brain Images}

The Midline shift (MLS) are the most important quantitative feature for clinician's to determine the severity of brain compression by various pathologies. The literature had proposed various models to detect Midline in Brain Images which is useful to calculate the asymmetry index of the brain for epileptic patients. Some examples of the literature for the deformed midline are by using the biomechanical properties of different types of intracranial tissue. The author [48] had presented an automated method to detect and classify an abnormality into acute infarct, chronic infarct and hemorrhage at the slice level of non-contrast CT images. The proposed method consists of three main steps: image enhancement, detection of mid-line symmetry and classification of abnormal slices. Hence by using the image registration and by detection of midline in the brain image helps to calculate the asymmetry index of the brain for the epileptic patients.

\subsubsection{Asymmetry Index Calculation}

An asymmetry index is obtained by placing regions of interest (ROIs) on PET image. The asymmetry indices technique is based on the assumption of having a relatively normal contralateral side and, thus, is not applicable in patients with bilateral abnormalities. Relative quantification is commonly achieved by count ratios which rely on the assumption of "normal" reference regions such as the visual cortex or cerebellum. Additionally, absolute quantification of regional glucose metabolism can be performed using prolonged dynamic acquisition and requires invasive arterial blood sampling [49-51]. When calculating the mean AI (Arterial Input), the absolute $\operatorname{AI}(=|\mathrm{AI}|)$ is used. The various approaches for PET are as follows:

In research scenario, the scans are assessed visually on two separate occasions by independent, experienced nuclear medicine physicians who are blinded to the clinical data. The inter-rater agreement is calculated. Semi quantitative analysis is performed, blind to the results of visual analysis, using a template of multiple $\mathrm{mm}$ circular ROIs placed in selected brain regions. For each structure, except the temporal poles, multiple ROIs were averaged. Asymmetry indices were calculated for homologous regions $[($ difference in uptake/average uptake $) \times 100 \%]$.

Asymmetry Index can also be calculated by using Wavelets. We propose a new solution to find asymmetric index of left and right hemisphere for brain data of any modality can be used. The Figure 4 shows the analysis of asymmetry index between left and right brain [52].

\subsubsection{Calculation for Inter Subject Asymmetric Variation}

To calculate the Inter subject variation of asymmetric index, the index is calculated for the model based left and right brain as the standard value, then we compare the real data with the model to find the inter subject asymmetric variation. The prior art for calculating asymmetry index is same as the intra subject calculation, such as medical image registration and the midline detection of the brain,

The increasing utilization of PET and 18FDG to assess normal brain function and its disruption in neuropsychiatries disorders brings us to evaluate normal variability of these measurements within a homogenous group of subjects [53]. The literature shows that in contrast to the low intrasubject variability, various studies have consistently reported large intersubject variability for absolute and relative measures of glucose metabolic rate in normal subjects at rest. Some of this variability may be accounted for by handedness, gender, mental status of the subject during the scan, brain volume and cortical atrophy and age.

Other researcher (Brain 2001) [54] has shown that intersubject asymmetry variability between the human percentral motor systems. The measurement of Brodmann area (BA 4) and the PRPT (Percentral component of the pyramidal tract) differ from the template of the Talairach atlas. They have used Morphological methods to show the significant correlation between the two and

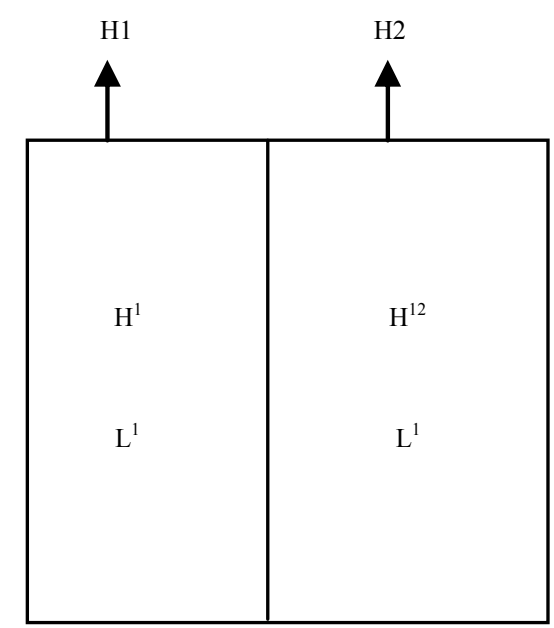

Figure 4. Asymmetric index using wavelets. 
also the variation in volumes. The literature shows that there are still research is going on to calculate the asymmetry index of the epileptic brain for the analysis of the brain model and with real left and right hemisphere [55].

\section{Conclusions \& Discussion}

Epilepsy research, including the development of innovative diagnostic and therapeutic interventions is ongoing. An epileptic seizure can be caused due to electric activity in any part of brain, and is analyzed through various techniques like EEG, MEG, physical examination, laboratory test or imaging system like PET, MRI, CT. Patient history and physical examination plays an important role to decide for further diagnostic tests and treatment. EEG a non-invasive device helps to identify any irregular electrical activity. This also helps to identify the zone of seizer. Neuro imaging is another area, which plays an important role in diagnosis and monitoring therapy response in epilepsy patient. This gives anatomical and functional information of brain. CT gives good anatomical details, whereas MRI gives good soft tissue contrast and bold imaging helps to identify functional details of brain. The combination of various imaging system helps to identify structural deformity and seizer location.

Nuclear scanner which includes PET is widely used for epileptic seizer detection and management of patients with seizure disorders. PET will continue to play a major role, not only in the clinical arena, but also in investigating the pathogenesis and treatment of various seizure disorders. There are various parameters analyzed on PET image data using various data processing tools for epileptic patients. These parameters includes cerebral blood flow measurement, uptake of fluorodeoxyglucose (FDG) an index of the cerebral metabolism rate of glucose, Distribution volume of flumazenil an index of the binding potential of central benzodiazepine receptor and standard uptake value. These parameters are analyzed by using asymmetry index within left and right hemisphere or comparing diseased parameters with standard values. The calculation of asymmetry index using PET is the significant technical parameters to find the amount of asymmetry in the left and right region of the brain hemisphere for the epileptic patients. It is always important to carry image registration and midline detection of brain before calculating asymmetry index in epileptic cases. Asymmetry index can be assessed using statistical analysis, soft computing approach, wavelet transform etc. The area of comparing left and right brain in the image data is not new, but analyzing asymmetry index is interesting and upcoming area to assess and localize disease area.

\section{Acknowledgements}

We thank the Philips Health Care for their support and encouragement, and providing us with datasets.

\section{REFERENCES}

[1] A. S. Fauci, E. Braunwald, D. L. Kasper, S. L. Hauser, D. L. Longo, J. L. Jameson and J. Loscalz, "Harrison Principles of Internal Medicine," 17th Edition, McGraw-Hill Professional, New York, 2008.

[2] N. Pillay, "Epilepsy: Medical and Surgical Approaches," Canadian Journal of Continuing Medical Education, 2001, pp. 224-232.

[3] M. Castillo, "Imaging Intractable Epilepsy: How Many Tests Are Enough?" American Journal of Neuro Radiiology, Vol. 20, No. 20, 1999, pp. 534-536.

[4] M. I. El-Gohary, A. S. A. Mohamed, M. M. Dahab, M. A. Ibrahim, A. A. El-Saied and H. A. Ayoub, "Diagnosis of Epilepsy by Artificial Neural Network," Journal of Biological Science, Vol. 8, No. 2, 2008, pp. 451-455. doi:10.3923/jbs.2008.451.455

[5] C. M. Verity, "The Place of the EEG and Imaging in the Management of Seizures," Archives of Disease in the Childhood, Vol. 73, No. 6, 1995, pp. 557-562.

doi:10.1136/adc.73.6.557

[6] C. M. Michela, M. M. Murraya, G. Lantza, S. Gonzaleza, L. Spinellib and R. G. de Peraltaa, "EEG Source Imaging," Clinical Neurophysiology, Vol. 115, No. 10, 2004, pp. 2195-2222.

[7] A. Hashizume, "Functional Brain Mapping and Localizing Epileptic Zone with Magneto Encephalography: A Review of Two Cases with Illustrations," Nepal Journal of Neuroscience, Vol. 4, 2007, pp. 106-109.

[8] R. G. de P. Menendez, S. L. G. Andino, M. S. C. M. Michel and T. Landis, "Imaging the Electrical Activity of the Brain: ELECTRA," Human Brain Mapping, Vol. 9, No. 1, 2000, pp. 1-12. doi:10.1002/(SICI)1097-0193(2000)9:1<1::AID-HBM1> 3.0.CO;2-\#

[9] J. Duncana, "The Current Status of Neuro Imaging for Epilepsy," Current Opinion in Neurology, Vol. 22, No. 2, 2009, pp. 179-184.

[10] T. M. Salmenpera and J. S. Duncan, "Imaging in Epilepsy," Journal of Neurology, Neurosurgery and Psychiatry, Vol. 76, No. 3, 2005, pp. 2-10.

[11] K. Engel, B. Bandelow, O. Gruber and D. Wedekind, "Neuroimaging in Anxiety Disorders," Journal of Neural Transmission, Vol. 116, No. 6, 2009, pp. 703-716. doi:10.1007/s00702-008-0077-9

[12] W. Dzienis1, E. Tarasów, J. Kochanowicz, A. Szulc, J. Walecki and B. Kubas, "Utility of Computed Tomography and Selected MR Sequences in the Diagnostics of Patients with Partial Epileptic Attacks," Medical Science Monitor, Vol. 13, No. 1, 2007, pp. 49-54.

[13] K. Chapman, E. Wyllie, I. Najm, P. Ruggieri, W. Bingaman, J. Luders, D. Dinner and H. O. Luders, "Seizure Outcome after Epilepsy Surgery in Patients with Normal Preoperative MRI," Journal of Neurology, Neurosurgery and Psychiatry, Vol. 76, No. 5, 2005, pp. 710-713. doi:10.1136/jnnp.2003.026757 
[14] S. Rastogi, C. Lee and N. Salamon, "Neuroimaging in Pediatric Epilepsy: A Multimodality Approach," Radiographics, Vol. 28, No. 4, 2008, pp. 1079-1095.

[15] E. Scharff, X. Papademetris, H. P. Hetherington, J. W. Pan, H. Zaveri, H. Blumenfeld, R. B. Duckrow, S. S. Spencer, D. D. Spencer, J. S. Duncan and E. J. Novotny, "Correlation of Magnetic Resonance Spectroscopic Imaging and Intracranial EEG Localization of Seizures," Proceedings of 3rd IEEE International Symposium on Biomedical Imaging: Nano to Macro, 6-9 April 2006, pp. 510-513.

[16] R. Sharma and R. Bargotra, "Magnetic Resonance Spectroscopy," JK Science, Vol. 8, No. 3, 2006, pp. 176-178.

[17] R. P. Lystad and H. Pollard, "Functional Neuroimaging: A Brief Overview and Feasibility for Use in Chiropractic Research," Journal of the Canadian Chiropractic Association, Vol. 53, No. 1, 2009, pp. 59-72.

[18] B. J. Casey, M. Davidson and B. Rosen, "Functional Magnetic Resonance Imaging: Basic Principles of and Application to Developmental Science," Developmental Science, Vol. 5, No. 3, 2002, pp. 301-309. doi:10.1111/1467-7687.00370

[19] A. Golby, D. Branco, S. Whalen and J. C. da Costa, "Functional MRI Memory Mapping for Epilepsy Surgery Planning: A Case Report," NeuroImage, Vol. 32, No. 2, 2006, pp. 592-602.

[20] R. C. Gonzalez and P. Wintz, "Digital Image Processing," Addison-Wesley, Boston, 1977.

[21] A. K. Jain and R. C. Dubes, "Algorithms for Clustering Data," Prentice-Hall, New York, 1988.

[22] M. Desco, J. D. Gispert, S. Sreig, A. Santos, J. Pascau, N. Malpica and B. P. Garcia, "Statistical Segmentation of Multidimensional Brain Datasets," SPIE Medical Imaging, Vol. 4322, No. 1, 2001, pp. 23-27.

[23] S. B. Mehta, C. Santanu, A. Bhattacharyya and A. Jena, "A Soft-Segmentation Visualization Scheme for Magnetic Resonance Images," Magnetic Resonance Imaging, Vol. 23, No. 7, 2005, pp. 817-828.

[24] S. B. Mehta, C. Santanu, A. Bhattacharyya and A. Jena, "Handcrafted Fuzzy Rules for Tissue Classification," Magnetic Resonance Imaging, Vol. 26, No. 6, 2008, pp. 815-823.

[25] S. B. Mehta, C. Santanu, A. Bhattacharyya and A. Jena, "Soft-Computing Based Diagnostic Tool for Analyzing Demyelination," Applied Soft Computing, Vol. 10, No. 2, 2010, pp. 529-538.

[26] K. Tina, W. E. L. Grimson, W. M. Well and R. Kikinis, "Segmentation of Brain Tissue from Magnetic Resonance Images," Medical Image Analysis, Vol. 1, No. 2, 1996, pp. 109-127. doi:10.1016/S1361-8415(96)80008-9

[27] P. A. Freeborough, N. C. Fox and R. I. Kitney, "Interactive Algorithm for the Segmentation and Quantization of 3D MRI Brain Scans," Computer Methods and Programs in Biomedicines, Vol. 53, No. 1, 1997, pp. 15-25.

[28] L. P. Clarke, R. P. Velthuizen, M. A. Camacho, J. J. Heine, M. Vaidyanathan, L. O. Hall, R. W. Thatcher and M. L. Silbiger, "MRI Segmentation: Methods Applications," Magnetic Resonance Imaging, Vol. 13, No. 3,
1995, pp. 343-368. doi:10.1016/0730-725X(94)00124-L

[29] M. Vermess, "Angiography in 'Idiopathic' Focal Epilepsy," Annual Journal of Radiology, Vol. 115, No. 1, 1972, pp. 121-128.

[30] R. Kuzniecky, "Clinical Applications of MR Spectroscopy in Epilepsy," Neuroimaging Clinics of North America, Vol. 14, No. 3, 2004, pp. 349-578.

[31] L. Wichert-Ana, A. C. Santos and P. M. A. de Marques, "SPECT and PET Imaging in Epilepsy: Principles and Clinical Applications," Journal of Epilepsy and Clinical Neurophysiology, Vol. 11, No. 1, 2005, pp. 19-30.

[32] R. Chu, "PET: An Emerging Technology," Proceedings of the Annual Symposium Computer Applied Medical Care, Vol. 1, 1980, pp. 469-475.

[33] L. Marinovich, "Positron Emission Tomography (PET) for Epilepsy," Commonwealth of Australia, 2005.

[34] A. Louca, "Imaging Tumoral Proliferation with Positron Emission Tomography (PET)," Journal of Applied Sciences Research, Vol. 4, No. 11, 2008, pp. 1337-1343.

[35] J. Blin, J. C. Baron, H. Cambon, A. M. Bonnett, B. Dubois and C. Loch, "Striatal Dopamine D2 Receptors in Tardive Dyskinesia: PET Study," Journal of Neurology, Neurosurgery, and Psychiatry, Vol. 52, No. 11, 1989, pp. 1248-1252. doi:10.1136/jnnp.52.11.1248

[36] W. K. Evans, "Implementing of PET Imaging According to Evidencebased Guidelines in Ontario", PET Steering Committee, Vol. 2, 2008, pp. 1-7.

[37] M. J. Koepp, C. Labbe, M. P. Richardson, D. J. Brooks, W. Van Paesschen and V. J. Cunningham, "Regional Hippocampal [11C] Flumazenil PET in Temporal Lobe Epilepsy with Unilateral and Bilateral Hippocampal Sclerosis," Brain, Vol. 120, No. 10, 1997, pp. 1865-1876. doi:10.1093/brain/120.10.1865

[38] K. Herholz and W.-D. Heiss, "Positron Emission Tomography in Clinical Neurology," Molecular Imaging \& Biology, Vol. 6, No. 4, 2004, pp. 239-269. doi:10.1016/j.mibio.2004.05.002

[39] J. S. Duncan, "Imaging and Epilepsy," Brain, Vol. 120, No. 2, 1997, pp. 339-377. doi:10.1093/brain/120.2.339

[40] W. H. Theodore, R. Brooks and R. Margolin, "Positron Emission Tomography in Generalized Seizures," Neurology, Vol. 35, No. 5, 1985, pp. 684-690. doi:10.1212/WNL.35.5.684

[41] B. Andrew, "PET in Seizure Disorders," Clinical Review, Vol. 43, No. 1, 2005, pp. 79-92.

[42] Y. Ohta, T. Nariai and K. Ishi, "Voxel- and ROI-Based Statistical Analyses of PET Parameters for Guidance in the Surgical Treatment of Intractable Mesial Temporal Lobe Epilepsy," Annual Nuclear Medicine, Vol. 22, No. 6, 2008, pp. 495-503. doi:10.1007/s12149-008-0140-5

[43] K. Ishiwata, K. Ishii, Y. Kimura, K. Kawamura, K. Oda, T. Sasaki, M. Sakata and M. Senda, "Successive Positron Emission Tomography Measurement of Cerebral Blood Flow and Neuro Receptors in the Human Brain: An 11C-SA4503 Study," Annual of Nuclear Medicine, Vol. 22, No. 5, 2008, pp. 411-416.

[44] J. S. Lee, D. S. Lee, S.-K. Kim, S. K. Lee, J.-K. Chung, M. C. Lee and K. S. Park, "Localization of Epileptogenic 
Zones in F-18 FDG Brain PET of Patients with Temporal Lobe Epilepsy Using Artificial Neural Network," IEEE Transactions on Medical Imaging, Vol. 19, No. 4, 2000, pp. 347-355. doi:10.1109/42.848185

[45] J. Thie, "Understanding the Standardized Uptake Value, Its Methods, and Implications for Usage," Journal of $\mathrm{Nu}$ clear Medicine, Vol. 45, No. 9, pp. 1431-1434.

[46] N. Adachi, M. Koutroumanidis, R. D. C. Elwes, C. E. Polkey and C. D. Binnie, "Interictal 18FDG PET Findings in Temporal Lobe Epilepsy With Deja vu," Journal of Neuropsychiatry Clinical Neuroscience, Vol. 11, No. 3, 1999, pp. 380-386.

[47] L. Junck, J. G. Moen, G. D. Hutchins, M. B. Brown and D. E. Kuhi, "Correlation Methods for the Centering, Rotation, and Alignment of Functional Brain Images," The Journal of Nuclear Medicine, Vol. 31, No. 7, pp. 12201226.

[48] C.-C. Liao, I.-J. Chiang and J.-M. Wong, "Tracing the Deformed Midline on Brain CT," Biomedical Engineering: Applications, Basis and Communications, Vol. 18, No. 6, 2006, pp. 305-311.

[49] K. W. Kang, D. S. Lee, J. H. Cho, J. S. Lee, J. S. Yeo, S. K. Lee, J.-K. Chung and M. C. Lee, "Quantification of F-18 FDG PET Images in Temporal Lobe Epilepsy Patients Using Probabilistic Brain Atlas," NeuroImage, Vol. 14, No. 1, 2001, pp. 1-6. doi:10.1006/nimg.2001.0783

[50] E. Luders, C. Gaser, L. Jancke, and G. Schlaug, "A Voxel-Based Approach to Gray Matter Asymmetries,"
NeuroImage, Vol. 22, No. 2, 2004, pp. 656-664. doi:10.1016/j.neuroimage.2004.01.032

[51] I. Yanovsky, P. M. Thompson, S. Osher and A. D. Leow, "Asymmetric and Symmetric Unbiased Image registration: Statistical Assessment of Performance," IEEE Computer Society Conference on Computer Vision and Pattern Recognition Workshops, Anchorage, 23-28 June 2008, pp. $1-8$.

[52] Y. K. Kumar and S. Mehta, "Asymmetry Analysis through Wavelet Transform in Brain Data," 2010. http://priorartdatabase.com/IPCOM/000191413

[53] G.-J. Wang, N. D. Volkow, A. P. Wolf, J. D. Brodie and R. J. Hitzemann, "Intersubject Variability of Brain Glucose Metabolic Measurements in Young Normal Males," The Journal of Nuclear Medicine, Vol. 35, No. 9, 1994, pp. 1457-1466.

[54] J. Rademacher and U. Burgel, "Variability and Asymmetry in the Human Percentral Motor System: A Cytoarchitectonic and Myoloarchitectonic Brain Mapping Study," Journal of Neurology, Vol. 124, No. 11, 2001, pp. 22322258.

[55] Y. Ohta, T. Nariai, K. Ishii, K. Ishiwata, M. Mishina, M. Senda, K. Hirakawa and K. Ohno, "Voxel- and ROIBased Statistical Analyses of PET Parameters for Guidance in the Surgical Treatment of Intractable Mesial Temporal Lobe Epilepsy," Annual Nuclear Medical, Vol. 22, No. 6, 2008, pp. 495-503. doi:10.1007/s12149-008-0140-5 